\title{
Parameter Modeling for Single Screw Pump Based On CATIA Secondary Development Platform
}

\author{
Heng $\mathrm{Fu}^{1, \mathrm{a}}$, Yanhua $\mathrm{Gu}^{2, \mathrm{~b}} *$, Xiaoyu Wang ${ }^{3, \mathrm{~b}}$, Xiu Fang Zhang ${ }^{4}$ \\ ${ }^{1,2,3}$ The School of Mechanical Science and Engineering, Jilin University ,Changchun, China \\ ${ }^{4}$ Jilin Agricultural Science and Technology University, Changchun, China \\ afuheng13@foxmail.com, ${ }^{b} 18143093360 @ 163 . c o m,{ }^{c}$ 307518066@qq.com
}

Keywords: Parameter modeling, Secondary development platform, CATIA, Single screw pump, VB Abstract. This article proposes parameter modeling for single screw pump and secondary development platform based on CATIA. First, we establish geometric parameter modeling of screw (rotor) and fixed bushing (stator) in CATIA. Then, a secondary development platform is developed by technology of VB and function of V5 Automation which is the primary secondary development platform for CATIA. Finally, we create a 3D model of 5/6 single screw pump and demonstrate the model's accuracy. The secondary development platform in the research can be applied to establish automatically 3D model of single screw pump, and facilitate the deep analysis and research for single screw pump.

\section{Introduction}

The screw pump which is essential component of a wide variety of engineering systems has been used in each area, especially petroleum field. Among various screw pumps, single screw pump is featured by small hydraulic pulse, abundant choices of transferring medium, large transferring capability and high stability and dependability of operations. Compared with piston pump and gear pump, it has huge advantages such as high pump pressure, large displacement, high efficiency and low consumption of energy [1]. So, as a result, researchers from international and domestic have deeply researched on single screw pump from different perspectives. V.N.Sarakuz presented modernized single screw pump [2]. Shatrov investigated design and technological features of shaping of single screw pump rotor profile [3]. Bereaux.Y proposed a simple model of throughput and pressure development for single pump [4].Jun Fei Wu presented effect of medium viscosity on the lift performance of full-metal single screw pump [5].

Many researches have been proposed as review above, but the process of modeling of single screw pump has taken much time. In order to increase efficiency, it is necessary to automatically generate modeling for single screw pump. The object of this present research is thus to tackle this issue by developing a CATIA secondary development platform. We establish geometric parameter modeling of screw (rotor) and fixed bushing (stator) in CATIA. Then, the rotor and stator are assembled to form a single screw pump. And by using CATIA V5 Automation API and VB technology, we develop the CATIA secondary development platform to automatically complete modeling of single screw pump. By this way, it take less time to create a new and accurate modeling of single screw pump, and provide a convenient to deeply research on parameters of single screw pump. 


\section{Rotor and stator geometric parameter equation}

At present, there are three types of single screw pump cycloid: the ordinary hypocycloid, the short-amplitude cycloid and the curtate epicycloid. The disadvantage of the ordinary hypocycloid and the curtate epicycloid is that it has some points which their curvature equal zero, so its equidistant curve exist the phenomenon of knot, which accelerate pump wear. As the result, we select the short-amplitude cycloid to design rotor and stator [6,7].

The short-amplitude cycloid stator equation

$$
\begin{aligned}
& x=\frac{z_{1} e}{k} \sin u-e \sin \left(z_{1} u\right) \\
& y=\frac{z_{1} e}{k} \cos u+e \cos \left(z_{1} u\right)
\end{aligned}
$$

Where, the variable $z_{1}$ is the number of rotor heads. The eccentricity e is a parameter that represents the distance between the moving point and the center of rolling. The variable $\mathrm{k}$ is the variable amplitude coefficient. The variable $\mathrm{u}$ represents the rolling angle.

The short-amplitude cycloid equidistance curve stator equation

$$
\begin{aligned}
& x_{1}=x-a \frac{y^{\prime}}{\sqrt{x^{\prime 2}+y^{\prime 2}}} \\
& y_{1}=y+a \frac{x^{\prime}}{\sqrt{x^{\prime 2}+y^{\prime 2}}}
\end{aligned}
$$

Where, the variable $a$ is the offset distance and $x$ is the derivative of $\mathrm{x}$ and $y$ ' is the derivative of $\mathrm{y}$.

The short-amplitude cycloid equidistance curve rotor equation

$$
\begin{aligned}
& x_{2}=x_{1} \cos \left(\frac{\varphi_{1}}{z_{1}+1}\right)-y_{1} \sin \left(\frac{\varphi_{1}}{z_{1}+1}\right)+a \sin \left(\frac{z_{1} \varphi_{1}}{z_{1}+1}\right) \\
& y_{2}=x_{1} \sin \left(\frac{\varphi_{1}}{z_{1}+1}\right)+y_{1} \cos \left(\frac{\varphi_{1}}{z_{1}+1}\right)+a \cos \left(\frac{z_{1} \varphi_{1}}{z_{1}+1}\right)
\end{aligned}
$$

Where, the variable $\varphi_{1}$ is the angle of two adjacent gears and the range of $u$ is between $-\frac{180^{\circ}}{z_{1}}$ and $\frac{180^{\circ}}{z_{1}}$.

\section{Parameter modeling for single screw pump}

Based on the equations of stator and rotor in the above, the modeling of single screw pump can be completed, through 3D design software CATIA. When building the 3D models for stator and rotor, the parameters of stator and rotor should be imported in the parametric modeling interface.

This article specifically introduces the process of the modeling of stator of $3 / 4$ single screw pump as follows. Firstly, in the formula window, the parameters which include $z_{1}, \mathrm{e}, \mathrm{k}$ and $\mathrm{u}$ are defined, and the result is showed in Figure1.Then, the equidistance curve equation of stator should be imported in Law Editor [8]. A point is established in the CATIA part design interface, through the equation of $x_{1}$ 
and the equation of $y_{1}$ in Law Editor. In order to generate the profile curve of stator, we use knowledgeware which is a part of CATIA to create some points which are connected to form the curve. Through user feature definition which contains features of the first point, we use the Loop Function to create the others points as shown in Figure 2 and Figure 3.

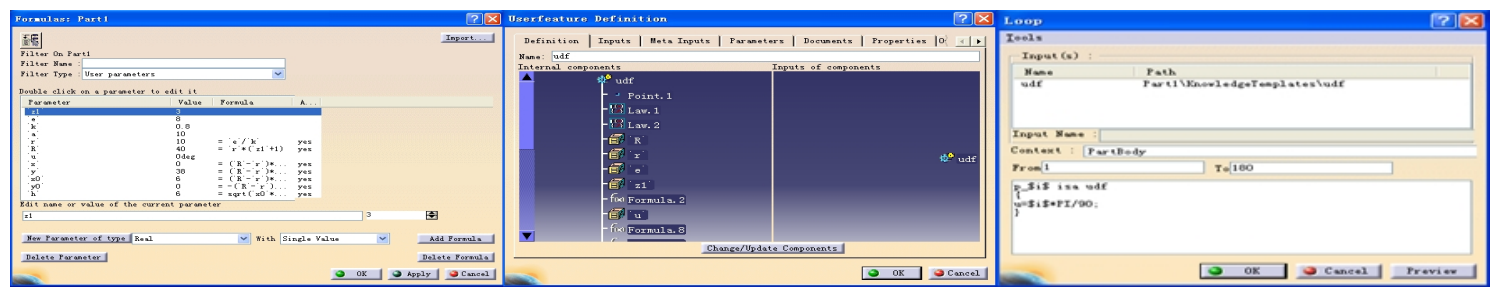

Figure1. Formula window

Figure 2. Userfeature definition

Figure 3. Loop

Then, the points are be connected by spline curve to form the profile of stator as shown in Figure 4.Finally, the 3D model of stator can be generated through the profile curve around the helix curve which is established along $\mathrm{z}$ axis as shown in Figure 5. With the same method, the 3Dmodel of rotor can be generated as shown in figure6, and the assembly model as shown in Figure 7.

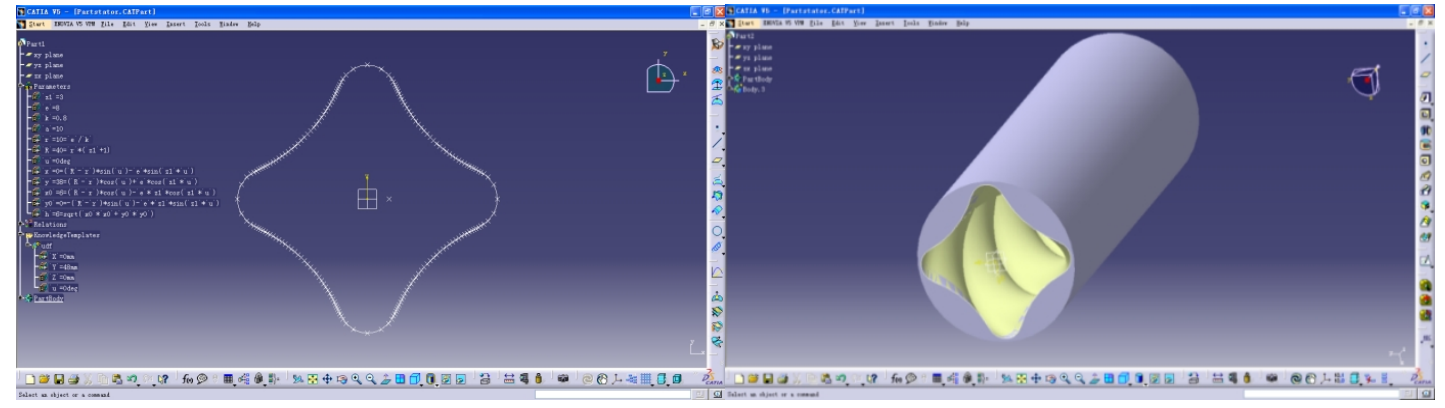

Figure 4. The profile curve of stator

Figure 5. The model of stator of $3 / 4$ single screw pump

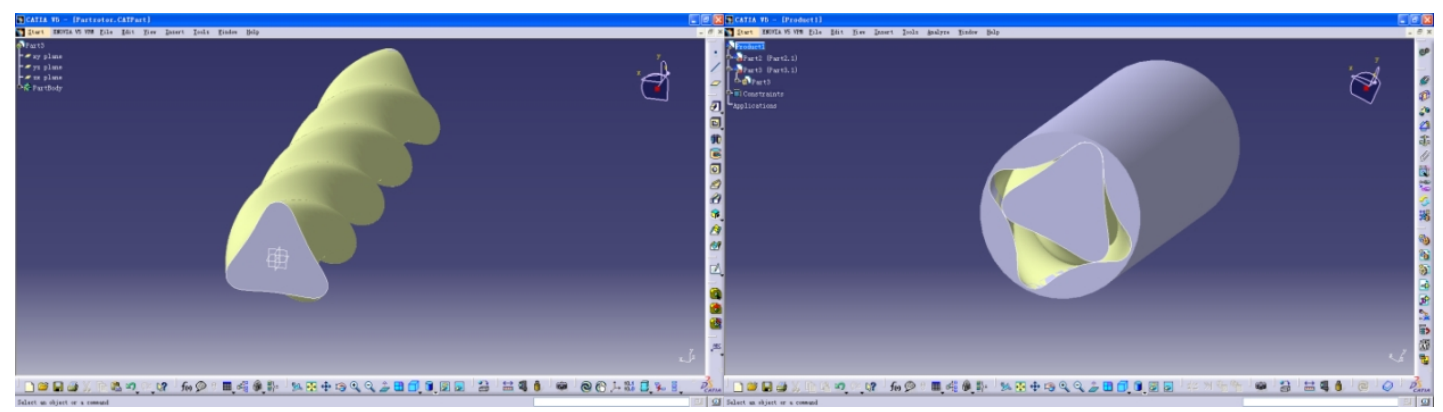

Figure 6 . The model of rotorof $3 / 4$ single screw pump Figure7. The assembly model of $3 / 4$ single screw pump

\section{CATIA secondary development platform}

The profile curve of stator and rotor of single screw pump is always complicated, so it takes much time to establish parameter model, and increases workload of researchers. In order to establish parameter model of single screw pump quickly and accurately, we develop the CATIA secondary development platform to automatically complete modeling of single screw pump by using CATIA Automation API and VB technology [9]. Some codes of the model of stator of 5/6 single screw pump as shown in Figure 8. 


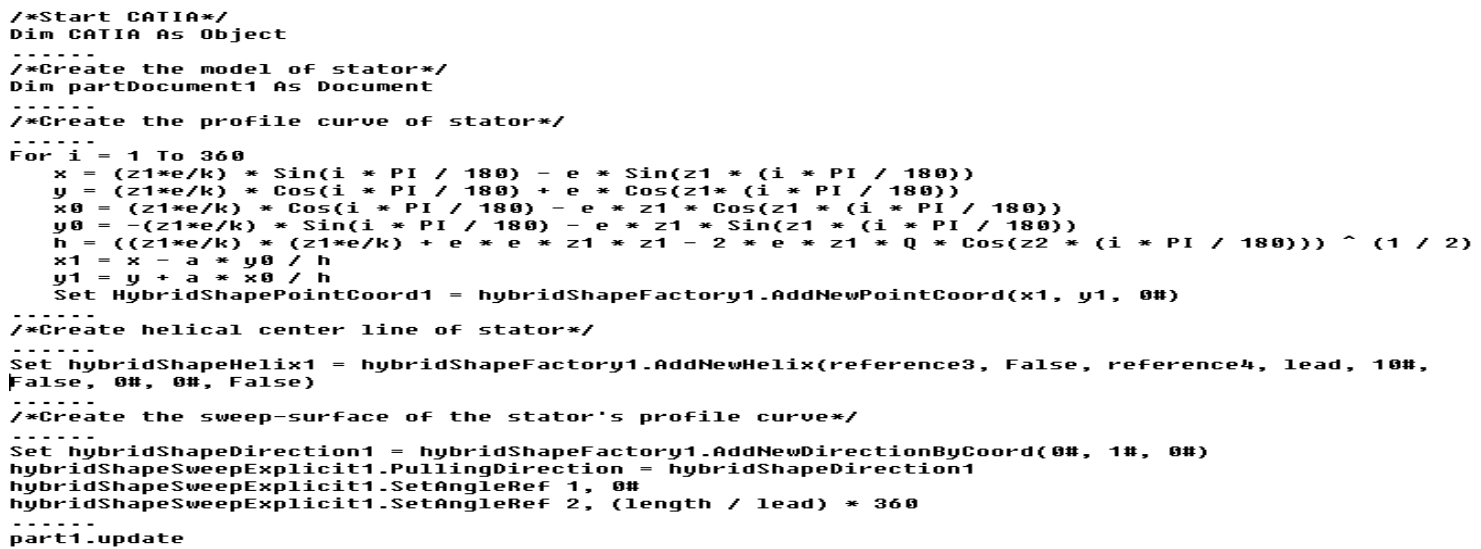

Figure 8. Some codes of the model of stator

The interface of VB for modeling of stator is shown in Figure9, and the modeling of stator is illustrated in Figure 10.

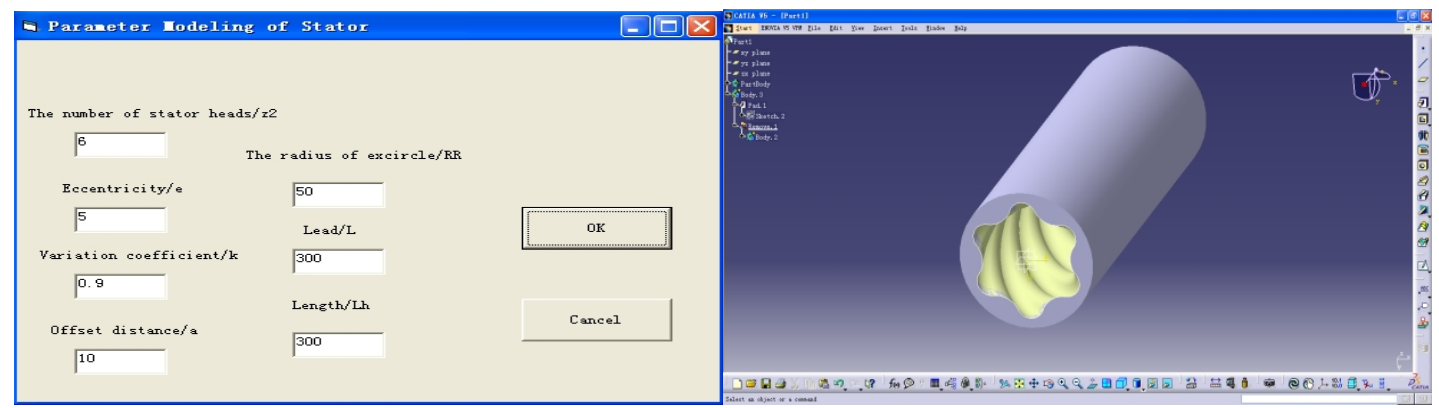

Figure 9. Parameter Modeling of Stator

Figure 10. The model of statorof $5 / 6$ single screw pump

The interface of VB for modeling of rotor is shown in Figure11, and the modeling of stator is illustrated in Figure 12.

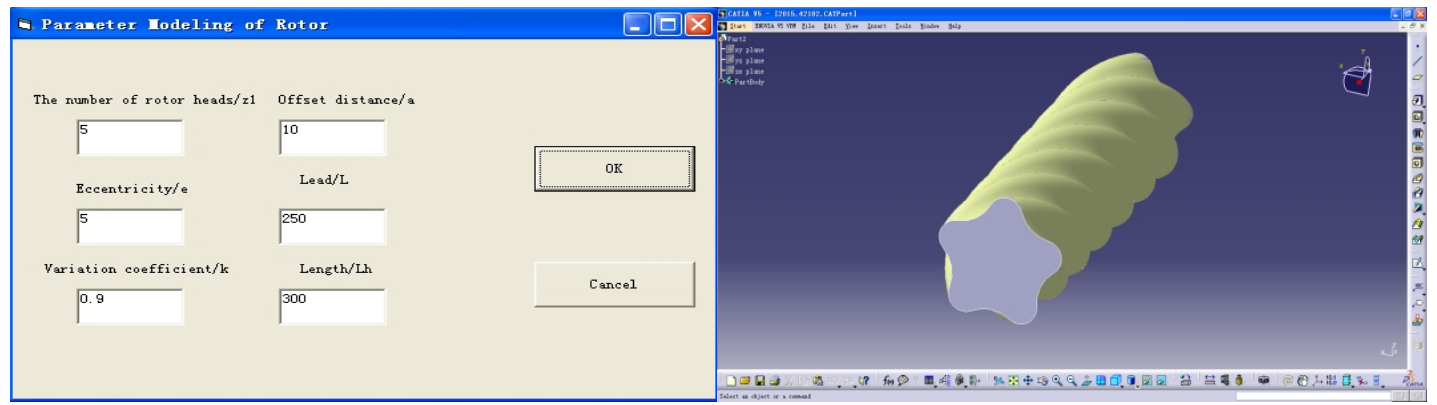

Figure 11. Parameter Modeling of Rotor

Figure12. The model of rotor of single screw pump

The assembly model of 5/6 single screw pump is shown in Figure 13.There is no interference in the assembly model which is tested by DMU Space Analysis function as illustrated in Figure 14. 


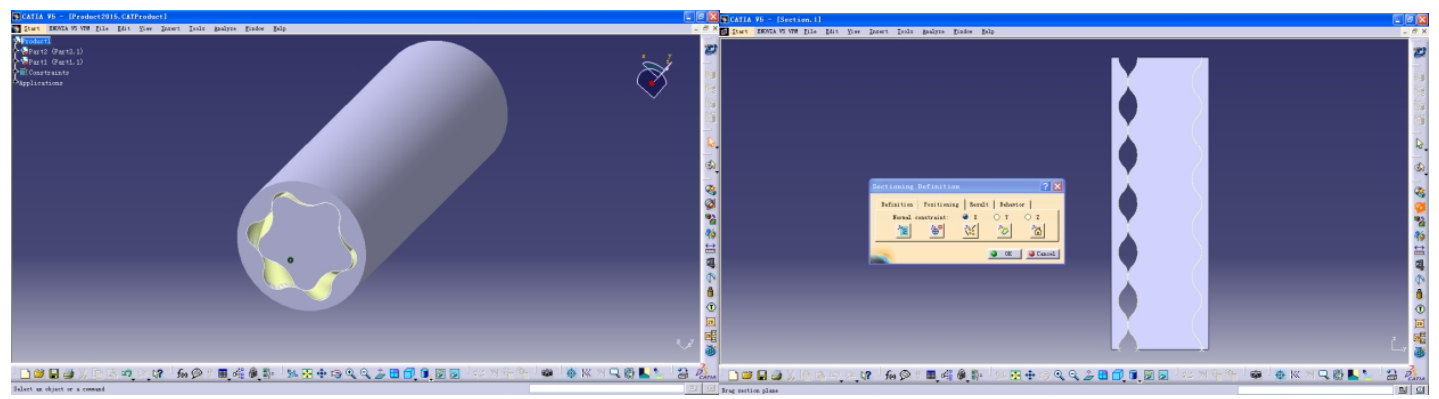

Figure 13. The assembly model of 5/6 single screw pump Figure 14 . Cutaway view from the direction of $\mathrm{x}$ axis

\section{Conclusions}

In the article, the implement of parameter modeling for rotor and stator based on CATIA is presented. Firstly, the mathematical parametric equations of rotor and stator are deduced which are then translated into the form adapted for the Law Function CATIA. Then, a model of 3/4 single screw pump is established in the modular of Knowledge Advisor in CATIA whose process is described briefly. Finally, based on the method of parameter modeling, the secondary development platform is established to automatically generate different typical kinds of single screw pump. A model of 5/6 single screw pump is quickly and accurately built by the platform. To validate the accuracy of the model, the interference is analyzed in the modular of DMU in CATIA. The secondary development platform greatly facilitates researchers developing the analysis of CAE (Computer-aided Engineering) and CAO (Computer-aided Optimization).

\section{References}

[1] Qian Tang and Yuanxun Zhang. Screw optimization for performance enhancement of a twin-screw pump. Proceedings of the Institution of Mechanical Engineers, Part E: Journal of Process Mechanical Engineering, 2014 ,pp.228: 73.

[2] V.N.Sarakuz, G. M. Timan'kov, V. I. Blokhin and V. N. Fraishtadt. Modernized single-screw pump. Chemical and Petroleum Engineering, 1982 ,Vol.18, NO.10, pp.447-448.

[3] Shatrov,VV;Gorshkov,GN;Lur'e,AI;Zal'tsberg,VK;Chadov, AV. Design and technological features of shaping of single-screw pump rotor profile. Chemical and Petroleum Engineering, 1999 , Vol.35, No.11-12, pp. 791-793.

[4] Béreaux,Y.;Charmeau,J.-Y.;Moguedet,M. A simple model of through put and pressure development for single screw. Journal of Materials Processing Technology, 2009, Vol.209, No.1, pp. 611-618.

[5] Jun Fei Wu* , Jin Mei Kan, Fan Guo Meng, Ben Liang Yu. Effect of Medium Viscosity on the Lift Performance of Full-Metal Single Screw Pump.Applied Mechanics and Materials, 2014, Vol.703, pp. 417-420.

[6] HAN Guo-you,REN Bin, DU Xiu-hua. Three heads single screw pump line design based onPro/E. Journal of DaQing Petroleum Institute.2006, Vol 30, No.4, pp. 49-51(In Chinese). 
[7] LIU Cheng-Lin, WANG Shi-jie. Linear design of long single-screw. Machinery Design \& Manufacture. 2010(1), pp. 36-38(In Chinese).

[8] YOU Chun-feng. CATIA V5 Advanced Application. Tsinghua University Press, 2006(In Chinese).

[9] HU Ting, WU Li-jun. Basic technology of CATIA secondary development. Electronic Industry Press. Bei Jing, 2006 (In Chinese). 